\title{
Reduction of divertor heat load in JET ELMy H-modes using impurity seeding techniques
}

\author{
J. Rapp ${ }^{1,9}$, P. Monier-Garbet ${ }^{2}$, G.F. Matthews ${ }^{3}$, R. Sartori ${ }^{4}$, \\ P. Andrew ${ }^{3}$, P. Dumortier ${ }^{5}$, T. Eich $^{6}$, W. Fundamenski ${ }^{3}$, \\ M. von Hellermann ${ }^{7}$, J. Hogan ${ }^{8}$, L.C. Ingesson ${ }^{7}$, S. Jachmich ${ }^{5}$, \\ H.R. Koslowski ${ }^{1}$, A. Loarte ${ }^{4}$, G. Maddison ${ }^{3}$, D.C. McDonald ${ }^{3}$, \\ A. Messiaen ${ }^{5}$, J. Ongena ${ }^{5}$, V. Parail ${ }^{3}$, V. Philipps ${ }^{1}$, G. Saibene ${ }^{4}$, \\ B. Unterberg ${ }^{1}$ and JET EFDA Contributors ${ }^{\mathrm{a}}$

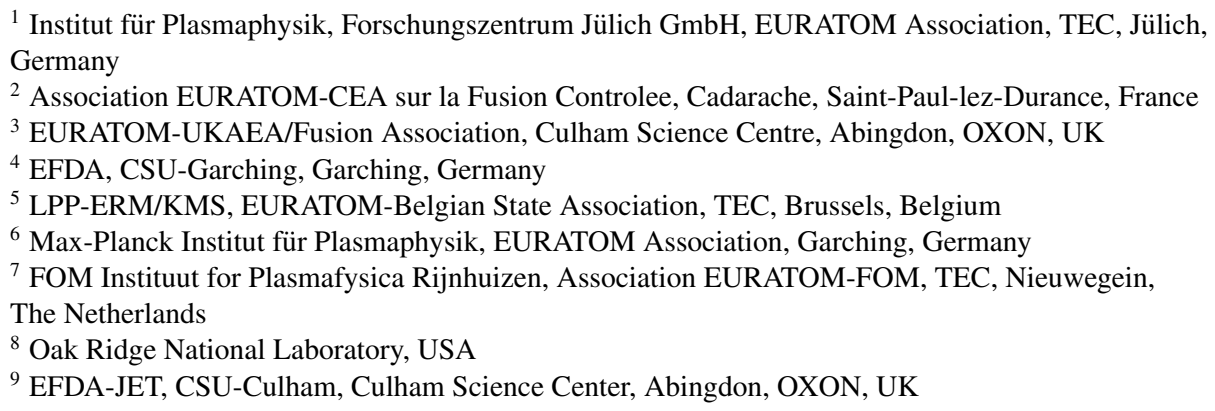

Received 12 March 2003, accepted for publication 5 December 2003

Published 16 January 2004

Online at stacks.iop.org/NF/44/312 (DOI: 10.1088/0029-5515/44/2/013)

\begin{abstract}
The main objective of this paper is investigation of methods for reduction of divertor heat loads in order to increase the lifetime of divertor tiles in future fusion reactors. Special emphasis is given to studies of reduction of transient heat loads due to edge localized modes (ELMs). Two methods are compared: argon seeded type-I ELMy H-modes and nitrogen seeded type-III ELMy H-modes. In both scenarios, the impurity seeding leads to a reduction in the pedestal energy and hence a reduction in the energy released by the ELM. This consequentially reduces the power load to the divertor targets. At high radiative power fractions in type-III ELMy H-modes, part of that released ELM energy (small ELMs, below $20 \mathrm{~kJ}$ ) is dissipated by radiation in the scrape off layer (SOL). Modelling of the ELM mitigation supports the experimental findings. This ELM mitigation by radiative dissipation is not effective for larger ELMs. In between ELMs, the plasma is detached and radiates strongly from the X-point region. During an ELM, the nitrogen in the X-point and divertor region becomes ionized into more weakly radiating higher charge states and the plasma re-attaches for large ELMs. At JET, argon radiates predominantly in the main plasma and not so much in the cold divertor region. Hence, the effect of radiative dissipation of ELM heat fluxes by argon is very low due to the limited argon density in the divertor region. Nevertheless, both scenarios might be compatible with an integrated ITER scenario, with respect to acceptable divertor lifetime and acceptable confinement.
\end{abstract}

PACS numbers: 52.55.Fu, 52.55.Rk, 52.25.Vy

\section{Introduction}

One of the most severe problems for fusion reactors is the power load to the divertor target plates. Technically, only

\footnotetext{
a See appendix of the paper 'Overview of recent JET results and future perspectives' by Pamela J. et al 2001 Proc. 18th Int. Conf. on Fusion Energy 2000 (Sorrento, 2000) (Vienna: IAEA).
}

steady state power loads of about $10 \mathrm{MW} \mathrm{m}^{-2}$ are acceptable, to allow sufficient margin for power excursions. In order to reduce the power load in the divertor to this value, radiation cooling by seeding of impurities might be necessary. For the ITER reference scenario a radiative power fraction of $\approx 75 \%$ is required [1]. Currently, in most fusion devices, carbon, which is the most commonly used divertor target and wall 
material, determines the radiative power fraction. Although some experiments achieved radiative power fractions of the order of $\approx 75 \%[2,3]$, at JET the radiative power fraction due to carbon seldom exceeds $50 \%$ without seeding of additional impurities. This is regarded as too marginal for the envisaged steady state power flux of $10 \mathrm{MW} \mathrm{m}^{-2}$ in ITER. However, looking even further ahead, especially to future devices with tungsten divertors and metallic walls (Be or $\mathrm{W}$ ), radiation due to intrinsic impurities will be minimal, and seeding of additional impurities becomes essential.

Furthermore, transient heat loads due to edge localized modes (ELMs) have to be reduced to values below $40 \mathrm{MJ} \mathrm{m}^{-2} \mathrm{~s}^{-1 / 2}$ (for a tungsten target). The current view is that unseeded type-I ELMy H-modes seem to be problematic. As the energy loss from the plasma due to ELMs, normalized to the pedestal energy, $\Delta W / W_{\text {ped }}$, increases with decreasing pedestal collisionality [4], the transient power load to the divertor target plates is expected to increase in a future device like ITER where the collisionality is reduced. For ITER, the estimated ablation limit and predicted heat load due to type-I ELMs are comparable [5]. Taking into account the uncertainty in the predictions with respect to the ELM loss in ITER and impact on the divertor target materials [6], it is prudent to develop alternative operating scenarios to the unseeded type-I ELMy H-mode with definite tolerable transient heat loads. One of the potential alternative scenarios is the ELMy H-mode with impurity seeding. Other potential solutions are the type-II ELMy regime and ELM mitigation by frequent pellet injection, which are not discussed in this paper. This paper summarizes the work aimed at reduction of the heat load to the target plates, both during and in between ELMs, using the radiation from an injected impurity. Two scenarios are described, type-I ELMy $\mathrm{H}$-modes with impurity seeding and type-III ELMy H-modes with impurity seeding.

\section{Radiative type-III ELMy H-modes}

In the first scenario described, nitrogen is seeded in type-III ELMy $\mathrm{H}$-modes up to radiative power fractions of $\approx 90 \%$. In low triangularity configurations [7], this regime leads to a partially detached $\mathrm{H}$-mode at $85 \%$ of the Greenwald density and confinement enhancement factors of $H_{98(y, 2)} \approx 0.7-0.85$ with a normalized plasma pressure of $\beta_{\mathrm{N}} \approx 1.3-1.7$.

\subsection{ELM buffering}

At high radiative power fractions, the steady state heat flux density is reduced from $8 \mathrm{MW} \mathrm{m}^{-2}$ to less than $1 \mathrm{MW} \mathrm{m}^{-2}$. By fuelling the plasma with deuterium and nitrogen, both the transient power load to the divertor due to ELMs and the transient energy loss due to ELMs from the plasma (at the pedestal) are decreased. At high radiative power fractions (90\%), the transient power loads due to ELMs are reduced to less than $5 \mathrm{MW} \mathrm{m}^{-2}$ at the outer divertor target plate [7] as measured by IR thermography and Langmuir probes. A reduction in the transient power loads to the divertor by increasing radiation does not necessarily mean that the ELM energy lost at the pedestal is dissipated by radiation in the scrape off layer (SOL) and/or divertor since the ELM energy loss, $\Delta W$, is reduced as well. A direct measurement of the energy loss from the plasma $(\Delta W)$ due to small type-III ELMs is difficult. For a few cases, kinetic measurements of the ELM energy loss were possible, which show at high radiative power fractions above $\approx 70 \%$ that the ELM energy loss from the plasma is around $25 \mathrm{~kJ}(\Delta W / W \approx 0.7 \%)$ [7].

However, an estimate of the energy loss can also be obtained from the power balance. In a steady state, the ELM energy loss is determined by the inter-ELM energy transport and the ELM frequency (for type-III ELMs): $P_{\text {in }}$ $\left(1-f_{\text {rad }}^{\text {inter-ELM }}\right) / f_{\mathrm{ELM}}=\Delta W+W_{\text {target, inter-ELM }}+W_{\text {wall, inter-ELM }}$. The energy flux to the wall, $W_{\text {wall, inter-ELM }}$, is negligible in between ELMs. In unseeded type-I ELMy H-modes, the interELM radiative power fraction (inter-ELM baseline) is usually of the order of $f_{\text {rad }}^{\text {inter-ELM }}=P_{\text {rad }}^{\text {inter-ELM }} / P_{\text {in }}=0.3-0.4$ (see also [7]). In the impurity seeded type-III ELMy H-modes, $f_{\text {rad }}^{\text {inter-ELM }}$ is increased, but an exact value cannot be given since the time resolution of the total radiated power measurements (bolometry, $20 \mathrm{~ms}$ ) allows only ELM averaged measurements of the radiated power. For partially detached type-III ELMy $\mathrm{H}$-modes (complete detachment in between ELMs), only a little inter-ELM power flux to the target is present. Hence, $\left(P_{\text {in }}-P_{\text {rad }}^{\text {inter-ELM }}\right) / f_{\text {ELM }}$ defines the maximum energy loss possible in an inter-ELM detached plasma. For pulse \#53318, shown in figure 1 , with $f_{\mathrm{ELM}}=300 \mathrm{~Hz}, P_{\text {in }}=11 \mathrm{MW}$ and taking as an estimate $f_{\text {rad }}^{\text {inter-ELM }}=0.3$, the maximum energy loss is $\Delta W \approx 26 \mathrm{~kJ}$. But for impurity seeded type-III ELMy H-mode discharges, $f_{\text {rad }}^{\text {inter-ELM }}$ will be higher (0.6-0.7), and thus the energy loss might be only $\Delta W \approx 10 \mathrm{~kJ}$. This has to

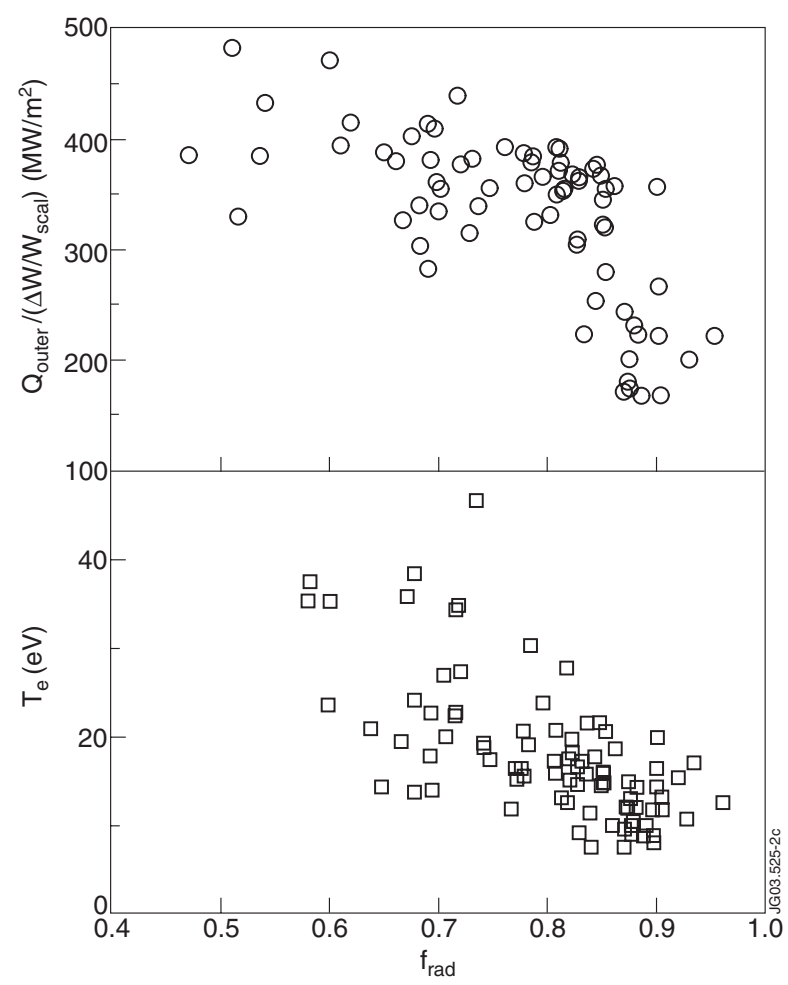

Figure 1. $\# 53318$, ratio of heat flux density, $Q_{\text {outer }}$, to the outer divertor target to the predicted normalized energy loss scaling, $\Delta W / W$-scaling $\left(\Delta W / W_{\text {scal }}=P\left(1-\mathrm{e}^{-b / f_{\text {ELM }} \tau_{E}}\right) / b P_{\text {in }}\right.$, see [27] $)$ and the electron temperature, $T_{\mathrm{e}}$, in front of the target during the ELM-peak (as measured by Langmuir probes versus the radiative power fraction. 
be compared with the measured value (kinetic measurements) of $25 \mathrm{~kJ}$ at a radiative power fraction of $70 \%$ (\#53772). The estimation above gives confidence in the kinetic measurements and is consistent with the fact that pulse \#53318 had a higher radiative power fraction, leading to lower ELM energy losses.

For these low ELM energies, the outer divertor plasma stays attached during the ELM energy pulse. This is different to the complete detached H-mode (CDH-mode) [8]. If the radiative power fraction is further increased up to $90 \%$, the electron temperature in front of the outer divertor, as measured by Langmuir probes, does not increase to more than $10-15 \mathrm{eV}$ on average during the ELM event. This is consistent with the radiation characteristic of nitrogen. The cooling rate has its maximum in the temperature range of $7-10 \mathrm{eV}$, and at higher temperatures $(10-30 \mathrm{eV})$ the cooling rate drops significantly (for corona equilibrium). This maximum of the cooling rate might be shifted slightly to higher temperatures in the presence of transport. However, nitrogen ions (Be- and Li-like ions) radiate most efficiently in the temperature range of $10-20 \mathrm{eV}$. For argon, this maximum (also here by Be- and Li-like ions) is at $\approx 300 \mathrm{eV}$. As a result, radiative dissipation of ELM energy is observed at radiative power fractions above $85 \%$, when the energy flux into the divertor is not sufficient to ionize the nitrogen in the divertor plasma to $\mathrm{He}$ - or H-like ions. Radiative dissipation in this sense means that with increasing radiative power fraction during ELMs the target heat flux is more strongly reduced than the energy loss from the plasma. This is shown in figure 1, where the ratio of the power density to the outer divertor target to the predicted ELM energy loss drops by a factor of more than 2. Although there might be an uncertainty in the absolute value of the ELM energy loss from the plasma (which is derived from a scaling, see [7] for details), the relative behaviour is important.

\subsection{Confinement and impurity behaviour}

Although the heat load can be reduced significantly, the confinement of these discharges is reduced as well. Typically type-III ELMy H-modes do have a 15-20\% lower confinement than type-I ELMy H-modes for comparable plasma densities (see figure 2). As the density and/or the radiation level is raised, the pedestal electron temperature and the pedestal ion temperature are decreased. The transition from type-I to type-III ELMy H-modes occurs when the pedestal temperature falls below a critical temperature [9]. Both pedestal and global confinement are lower in H-modes with type-III ELMs compared with H-modes with type-I ELMs. For more detailed studies on the transition from type-I to type-III ELMy H-modes with gas fuelling at constant power, see $[10,11]$. However, increasing the triangularity from $\delta=0.2$ to 0.47 leads to improved confinement at high densities generally for type-I and type-III ELMy H-modes [12]. In plasmas with these high triangularity configurations, the transition to type-III ELMy $\mathrm{H}$-modes at a high density is rather sharp, and it is characterized by a large increase in ELM frequency. A more gradual transition from one ELM regime to the other is observed in ELMy H-modes at low triangularity. In these plasmas, the pedestal and global confinement decrease continuously with increasing density and ELM frequency.

Figure 3 shows the dependence of $H_{98(y, 2)}$ for radiative type-III ELMy $\mathrm{H}$-modes with radiative power fractions of

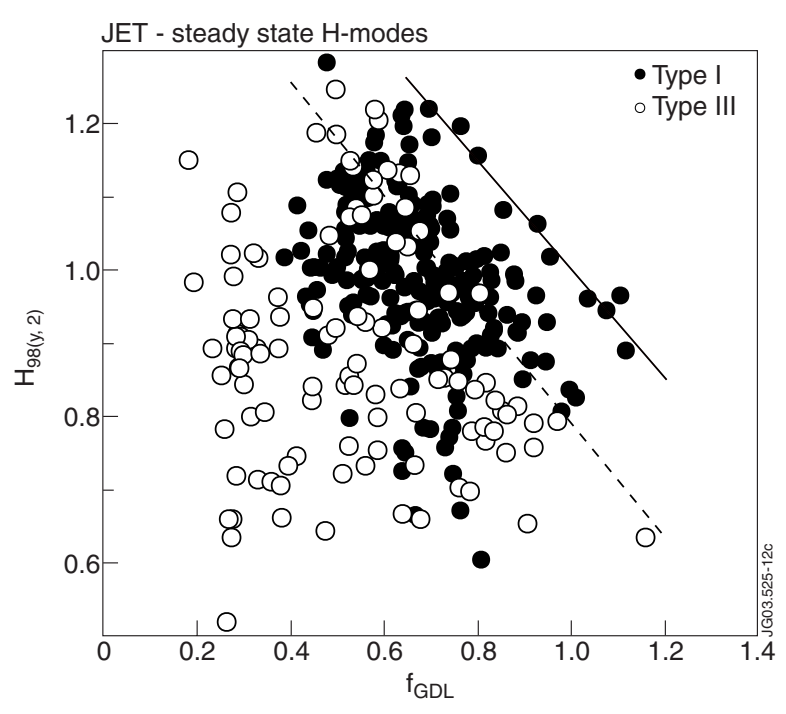

Figure 2. Confinement enhancement factor, $H_{98(y, 2)}$, versus the normalized density, $\bar{n}_{\mathrm{e}} / n^{\mathrm{GW}}=f_{\mathrm{GDL}}$, for unseeded type-I and type-III ELMy H-modes; steady state database.

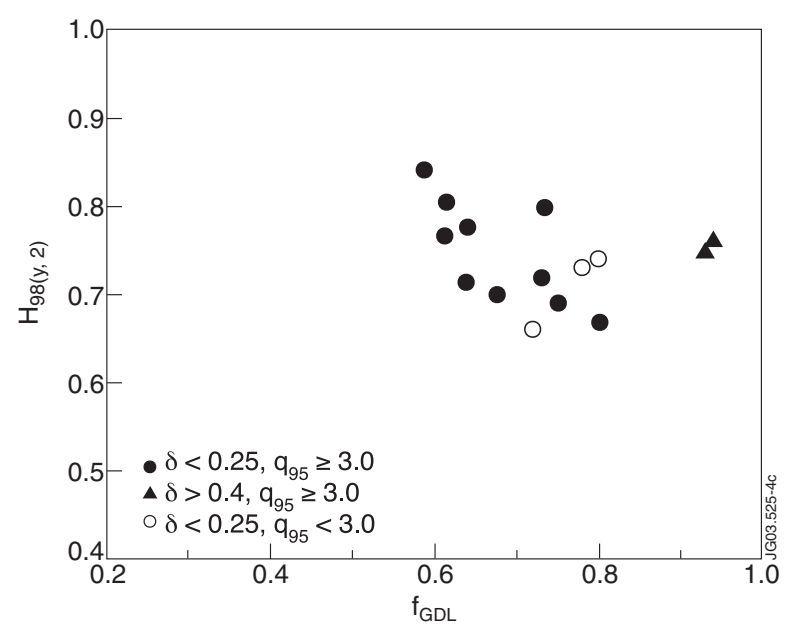

Figure 3. Confinement enhancement factor, $H_{98(y, 2)}$, versus the normalized density, $\bar{n}_{\mathrm{e}} / n^{\mathrm{GW}}=f_{\mathrm{GDL}}$, radiative type-III ELMy $\mathrm{H}$-modes with radiative power fraction $f_{\text {rad }} \geqslant 0.7$.

$70 \%$ and larger as a function of the plasma density. As observed in the unseeded discharges, a reasonable confinement $H_{98(y, 2)} \approx 0.75$ can be kept up to densities close to the Greenwald density, $\bar{n}_{\mathrm{e}} / n_{\mathrm{e}}^{\mathrm{GW}}=f_{\mathrm{GDL}}=1$. For low densities, high $Z_{\text {eff }}$ are observed. In low triangularity impurity seeded $\mathrm{H}$-modes, $Z_{\mathrm{eff}}$ is in the range of 2-2.5. An increase in absolute density $\left(Z_{\text {eff }} \propto P_{\text {rad }} / n_{\mathrm{e}}^{2}\right)$ leads to a reduction in the plasma core impurity content. The lowest $Z_{\text {eff }}(\approx 1.6)$ was achieved in high triangularity $2.5 \mathrm{MA} / 2.7 \mathrm{~T}$ pulses. No impurity accumulation has been observed. The profile of the fully ionized nitrogen, as derived from CXRS, is hollow during the highest radiative power fractions.

\subsection{ITER implications}

The radiating type-III ELMy H-mode described above could enable an integrated ITER scenario for $Q=10$ operation with acceptable steady state and transient target power loads. 
For a slightly degraded confinement $\left(H_{98(y, 2)}=0.75-0.8\right)$, $Q=10$ operation will be possible at a plasma current of $17 \mathrm{MA}$ $\left(\beta_{\mathrm{N}} \approx 1.5, f^{\mathrm{GDL}}=1, q_{95}=2.6\right)[13,1]$. This operational domain for $17 \mathrm{MA}$ is shown in figure 4 .

Operation at low $q_{95}$ may prove more difficult due to MHD activities, i.e. neoclassical tearing modes (NTMs) and locked modes. In JET, however, the operational domain at low triangularity and high radiative power fractions $\left(f_{\text {rad }} \geqslant 0.7\right)$ has been extended to low $q_{95}=2.6$ with no apparent drawback due to MHD activities. The confinement $\left(H_{98(y, 2)}=0.75\right)$ is just acceptable at low edge safety factors (see figure 5 ). Some reduction of the confinement enhancement factor $H_{98(y, 2)}$ at low edge safety factors is observed, and the reason for this is still under investigation. For NTM-free type-I ELMy $\mathrm{H}$-modes, no $q_{95}$ dependence of the confinement is observed at JET [14].

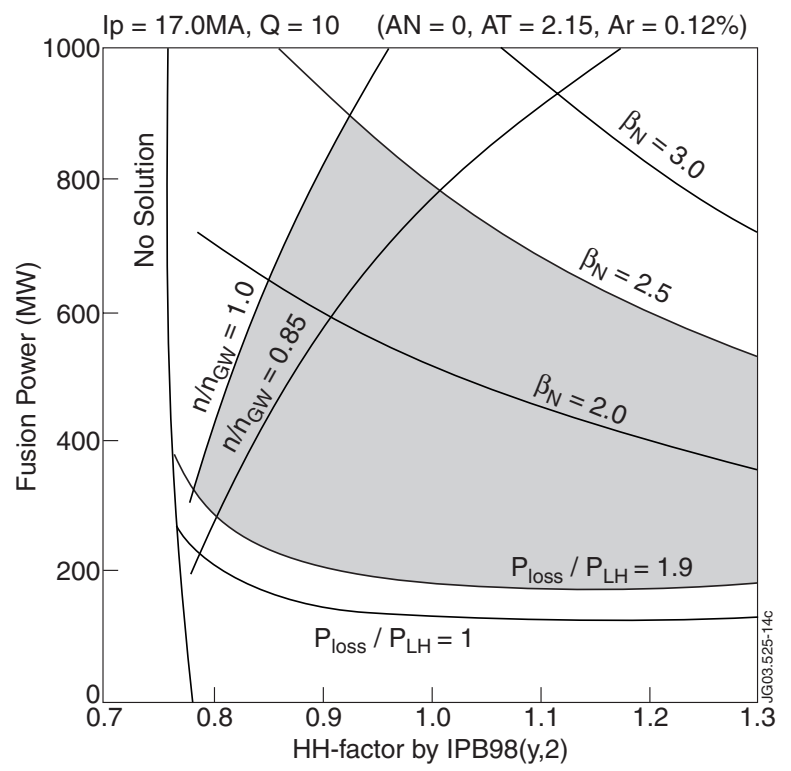

Figure 4. Fusion power versus the confinement enhancement factor, $H_{98(y, 2)}$; the $Q=10$ domain is indicated by the shaded area. For explanations of parameters, see [1] and references therein (the figure is courtesy of M. Shimada).

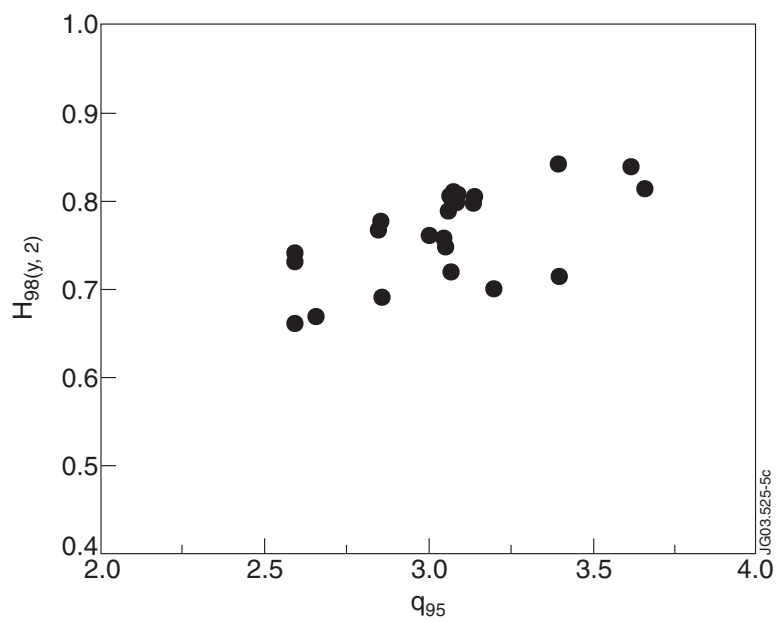

Figure 5. Confinement enhancement factor, $H_{98(y, 2)}$, versus the edge safety factor, $q_{95}$; radiative type-III ELMy H-modes with radiative power fractions $f_{\mathrm{rad}} \geqslant 0.7$ and triangularity $\delta \leqslant 0.25$.

\section{Radiative type-I ELMy H-modes}

As an alternative to the nitrogen seeded discharges, argon is seeded in type-IELMy H-modes in order to reduce the transient heat flux due to larger ELMs (energy losses due to ELMs of $\Delta W \geqslant 0.2 \mathrm{MJ}$ ) since it has the potential to radiate at higher temperatures. In this scenario, a confinement of $H_{98(y, 2)}=1$ can be maintained at densities of $f_{\mathrm{GDL}}=0.85$ up to radiative power fractions of $65 \%$ with a careful adjustment of both the argon and deuterium fuelling rates [15].

\subsection{ELM buffering}

In the series of experiments reported here, emphasis has been given to analysis of the ELM heat flux in discharges that have not been performance optimized. Performance optimized in this sense means that these discharges are not 'after-puff' experiments [15] and are not high triangularity configurations. In the experiments reported here, the key parameters are $H_{H 98(y, 2)} \approx 0.87, f_{\mathrm{GDL}}=0.78$ (which is $5 \%$ less than in the deuterium reference pulse) and $f_{\text {rad }}=0.6$. Detailed measurements of the pedestal parameters (edge LIDAR [16] and ECE data [17]) and of the power fluxes to the target plates (IR camera [18]) have been obtained. In these experiments, the frequency of the type-I ELMs decreases slightly as the power crossing the pedestal decreases, due to enhanced radiative power from the plasma core (see also [19]), and the transient divertor power load is reduced by a factor of $\approx 2$ (figure 6 ). The resulting peak value at the outer divertor target is $\approx 15 \mathrm{MW} \mathrm{m}^{-2}$, i.e. a factor of 3 higher when compared with the type-III ELMy H-mode with similar heating power. This reduced transient heat flux with argon seeding stems from a reduced pedestal electron temperature (figure 7) and hence, since the pedestal density was not changed, from a decreased pedestal energy and an increased pedestal collisionality. This is furthermore illustrated in

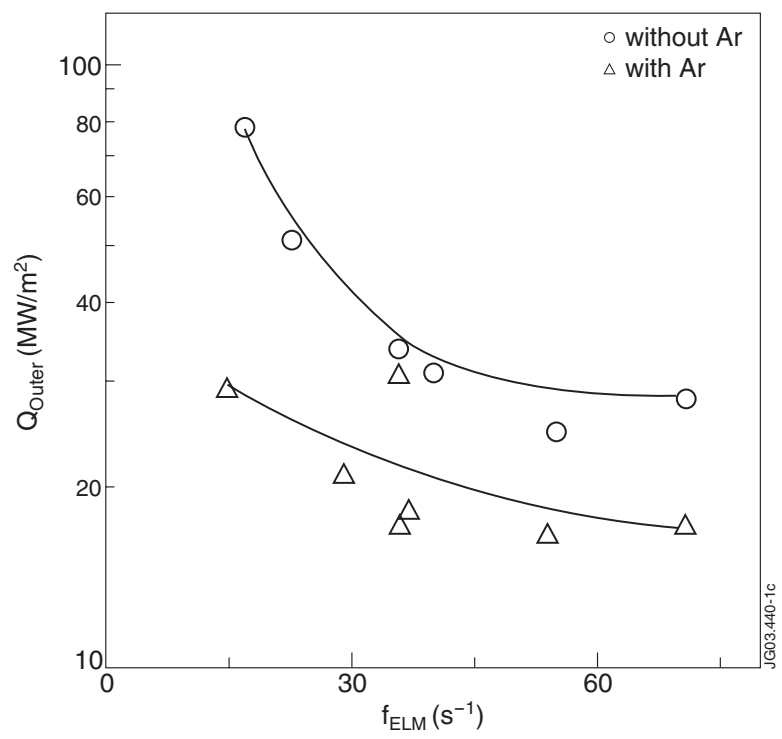

Figure 6. Comparison of peak heat fluxes (during ELM event) to the outer divertor target, $Q_{\text {outer }}$, with a radiative power fraction $\left(f_{\text {rad }} \approx 0.6\right)$ and without argon fuelling versus the ELM frequency, $f_{\text {ELM }}$. 


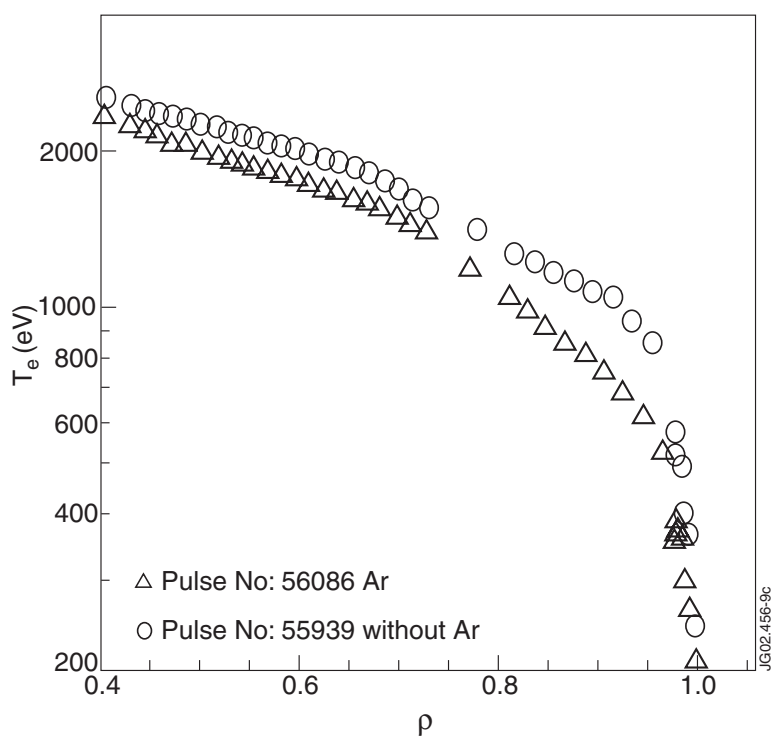

Figure 7. Comparison of the electron temperature profiles, $T_{\mathrm{e}}$, with and without argon seeding versus normalized plasma minor radius $\rho=r / a_{\min }$

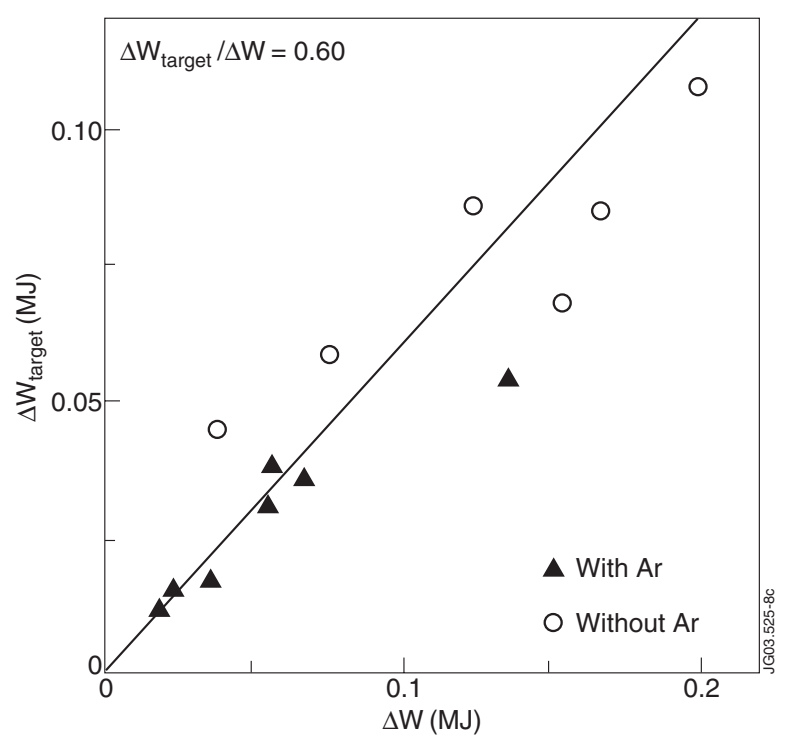

Figure 8. Target energy load due to ELMs, $\Delta W_{\text {target }}$, versus energy lost from plasma due to ELMs, $\Delta W$.

figure 8, which shows the energy deposited at the divertor target versus the loss of stored plasma energy: no difference between data from argon seeded and non-seeded discharges is observed, demonstrating the lack of additional dissipation in the SOL and divertor plasma under these conditions. However, for similar ELM losses, $\Delta W / W_{\text {ped }}$, argon seeded discharges have a lower ELM frequency (by up to a factor of 2 lower, see figure 9).

\subsection{ITER implications}

Argon seeding in type-I ELMy H-modes would enable an integrated ITER scenario for $Q=10$ operation at $15 \mathrm{MA}$ $\left(q_{95}=3\right)$. Confinement and density are well within the ITER requirements [20]. However, the aimed radiative power fraction of $f_{\text {rad }}=0.75$ is difficult to reach in this scenario.

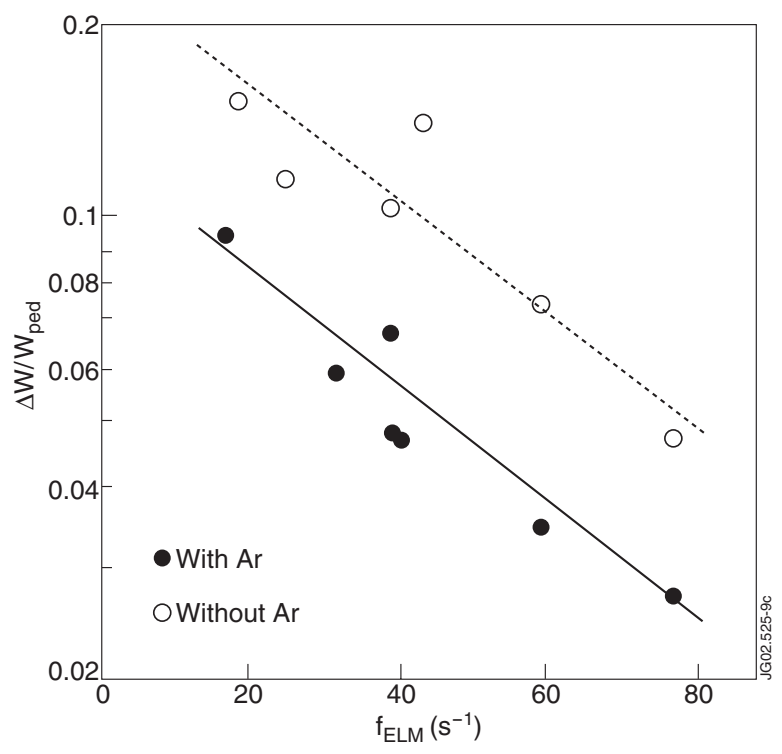

Figure 9. Dependence of normalized (to pedestal energy) ELM energy losses from plasma, $\Delta W / W_{\text {ped }}$, versus local ELM frequency, $f_{\mathrm{ELM}}$.

It is observed that the steady state heat flux to the divertor is not significantly reduced in these pulses, consistent with the modest rise in radiated power. The maximum radiative power fraction has to be limited to avoid a transition into a type-III ELMy H-mode and to avoid accumulation of argon in the plasma centre. At JET, it has been found that the maximum achievable radiative power fraction in stationary type-I ELMy $\mathrm{H}$-modes is $f_{\text {rad }}=0.65$. At higher radiative power fractions, the local core radiation power density exceeds the local heating power density and an accumulation instability on the basis of the neoclassical theory develops [21]. This has detrimental effects on the confinement [22], although central heating by ion cyclotron resonance heating (ICRH) might enable high impurity concentrations without accumulation in the plasma core [22]. This is due to the changed particle transport in the plasma core induced by ICRH.

\section{Modelling of radiative dissipation in the SOL by EDGE2D/NIMBUS}

In order to investigate the effect of radiative dissipation in highly radiating plasmas, multi-species time dependent EDGE2D/NIMBUS modelling has been carried out [23]. As a reference plasma, pulse \#53318 was taken with the Mk-II gas box divertor as boundary. The total heating power is $\approx 11 \mathrm{MW}$ with $8 \mathrm{MW}$ input power to the modelling grid, and $2 \mathrm{MW}$ is radiated in the plasma core. Nitrogen is puffed into the inner divertor in this simulation with a rate of $8 \times 10^{21}$ atoms s $^{-1}$, which is comparable with the experimental fuelling rate of $5 \times 10^{21}$ from \#53318. The radiative power fraction is $85 \%$, with nitrogen the main radiator $(60 \%$ of the total radiated power is from nitrogen and $40 \%$ from carbon). This target plasma is used as a basis to study the influence of the ELM energy on the target energy load. The ELM is simulated by an increased radial particle diffusion coefficient, an increased ion thermal diffusion coefficient and an increased electron thermal diffusion coefficient. The initial transport coefficient 
Reduction of divertor heat load in JET ELMy H-modes

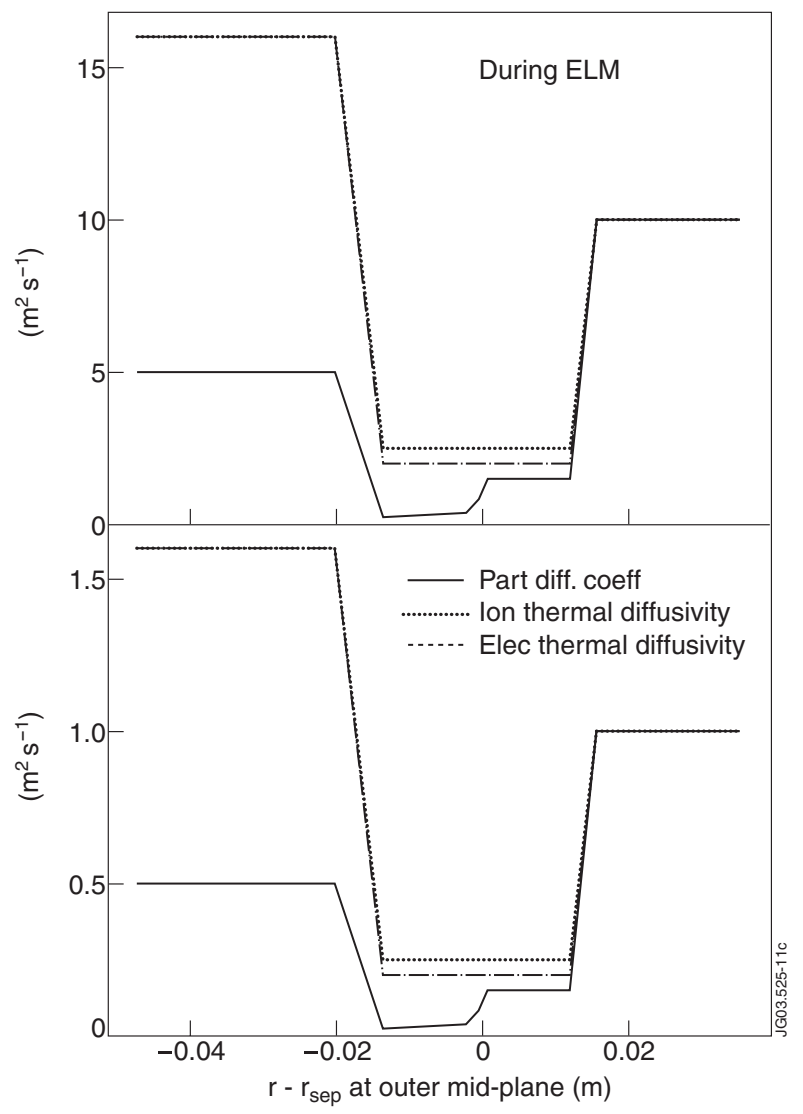

Figure 10. Radial profile of transport coefficients as used in EDGE2D/NIMBUS: particle diffusion coefficient, ion thermal diffusivity, electron thermal diffusivity versus radius in midplane relative to separatrix radial position; before (lower figure) and during a simulated $25 \mathrm{~kJ}$ ELM (upper figure).

profiles, which have been chosen to reproduce realistic pedestal electron temperature profiles [24], are multiplied by a constant factor. Typically, the particle diffusion coefficient is in the centre $\approx 0.5 \mathrm{~m}^{2} \mathrm{~s}^{-1}$ and in the SOL $\approx 1 \mathrm{~m}^{2} \mathrm{~s}^{-1}$. Around the pedestal region, the transport coefficients are reduced to much lower values (for details see [24]). Increasing the transport coefficients in EDGE2D by factors of 2.5, 5, 10 and 30 leads to ELM energies of 5, 10, 20 and $60 \mathrm{~kJ}$. The radial profile of the transport coefficients before and during the ELM are shown in figure 10. The duration of the increased transport is $0.5 \mathrm{~ms}$, which is consistent with the experimental ELM rise time. An example of such an ELM simulation is shown in figure 11. This modelling of the ELM mitigation demonstrates that a significant part of the ELM energy can only be dissipated by radiation for ELMs smaller than $20 \mathrm{~kJ}$ (see figure 12). Increasing the ELM energy to $60 \mathrm{~kJ}$ diminishes the effect of radiative dissipation in these highly radiative plasmas to negligible values of $\approx 20 \%$. The target energy load due to ELMs for the larger ELMs $(60 \mathrm{~kJ})$ is about $50 \%$ of the ELM energy loss from the plasma due to losses to the edge of the EDGE2D grid (mainwall), consistent with experimental observations (see above). Without the cooling by extrinsic impurities (here nitrogen), ELM mitigation of small ELMs $(25 \mathrm{~kJ})$ is even less effective ( $\leqslant 20 \%$ gets dissipated).

As observed in the experiment, the electron temperature in front of divertor targets does stay below $14 \mathrm{eV}$ (during the

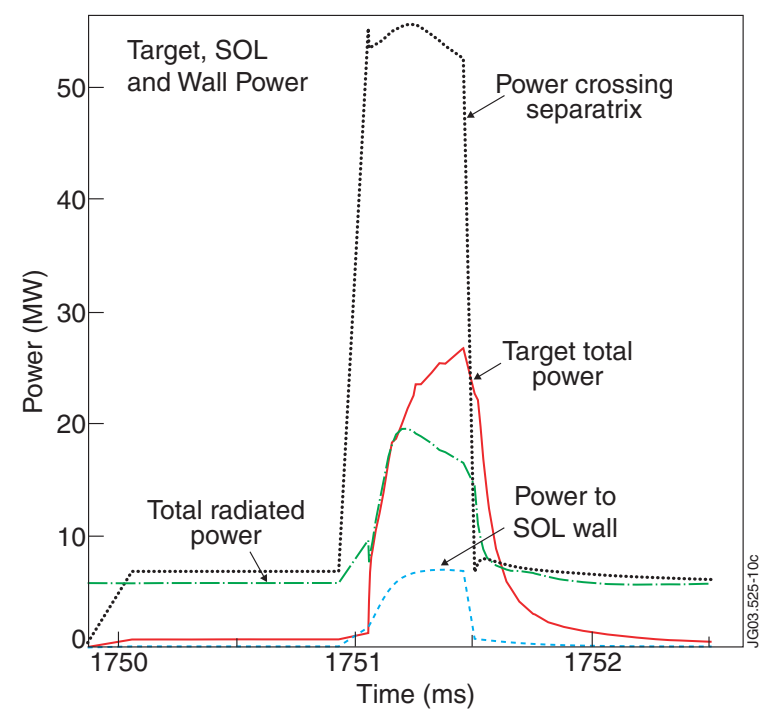

Figure 11. Example of an ELM simulation with

EDGE2D/NIMBUS, $25 \mathrm{~kJ}$ ELM: power crossing the separatrix, the total heat flux to the outer and inner divertor targets, the total radiated power, which includes radiation from nitrogen and carbon, heat flux to the wall (meaning cross field heat flux leaving computational grid). Simulation parameters: electron midplane separatrix density $n_{\mathrm{e}} \approx 2.0 \times 10^{19} \mathrm{~m}^{-3}$, midplane separatrix temperatures before ELM, $T_{\mathrm{e}}=80 \mathrm{eV}$, and $T_{\mathrm{i}}=190 \mathrm{eV}$, SOL width is $\lambda \approx 5 \mathrm{~mm}$ (consistent with experimental findings), flux-limited ion and electron parallel heat transport, drift effects are excluded.

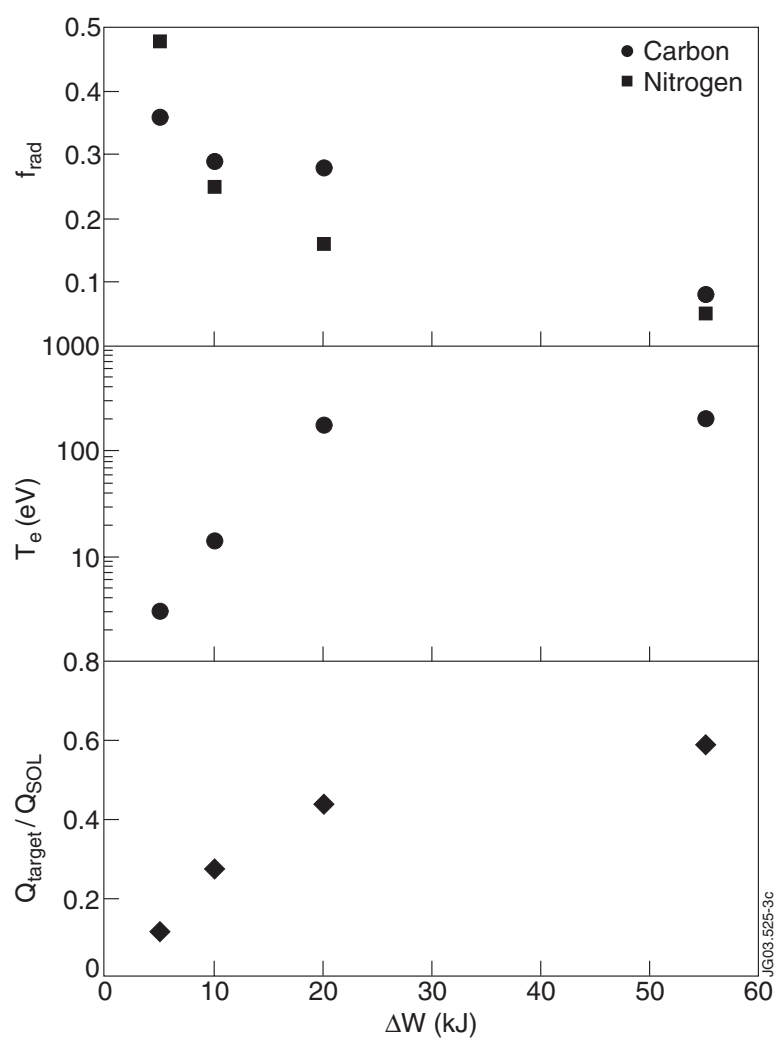

Figure 12. EDGE2D/NIMBUS results: radiative power fraction, $f_{\text {rad }}$, of nitrogen and carbon during ELM event, electron temperature, $T_{\mathrm{e}}$, in front of outer divertor target during ELM event and ratio of target heat flux to heat flux crossing the separatrix during ELM event versus the ELM stored energy loss. 
ELM peak) for ELM energies below $10 \mathrm{~kJ}$. For these small ELMs, nitrogen becomes the main radiator during the ELM heat flux, whereas for the larger ELMs $(\Delta W \geqslant 20 \mathrm{~kJ})$, carbon radiates stronger than nitrogen. The increased target fluxes during the ELM event lead to an increased carbon release and then subsequentially radiate part of the ELM energy.

The EDGE2D/NIMBUS results reported here for JET are consistent with B2/Eirene simulations for ITER [25], which show that only extremely small ELMs can be dissipated by a radiating layer. A comparison of recycling impurities versus non-recycling impurities has been carried out (1\% recycling versus $90 \%$ recycling), which shows essentially no significant difference in the effect of radiative dissipation.

\section{Discussion}

In order to assess the applicability of both impurity seeding scenarios to ITER, the expected ELM size in ITER has to be estimated. Figure 13 shows the dependence of the ELM energy loss on the pedestal collisionality. The pedestal collisionality is given by $\nu_{\text {ped, } \|}^{*}=\pi \times R \times q_{95} / \lambda_{\mathrm{e}, \mathrm{e}}$, with $\lambda_{\mathrm{e}, \mathrm{e}}=1.44 \times$ $10^{23} \times T_{\mathrm{e}}^{2} /\left(n_{\mathrm{e}} \times \ln \lambda\right), T_{\mathrm{e}}$ in $\mathrm{keV}, n_{\mathrm{e}}$ in $\mathrm{m}^{-3}$ and $\ln \lambda$ being the Coulomb logarithm. Both in type-I ELMy H-modes and in type-III ELMy H-modes, the energy loss due to ELMs, $\Delta W / W_{\text {ped }}$, increases with decreasing pedestal collisionality. Argon seeded type-I ELMy H-modes have the same ELM energy loss, $\Delta W / W_{\text {ped }}$, as unseeded type-IELMy H-modes for similar collisionalities. For type-III ELMy H-modes also, no difference between impurity seeded and non-seeded discharges with respect to the ELM energy losses is observed at similar collisionalities. One should note that the values of $\Delta W / W_{\text {ped }}$ used in figure 13 for type-III ELMs are upper boundary estimates of the real ELM losses since the noise level in the magnetic measurements is comparable with $\Delta W$. This upper estimate for the energy loss in type-III ELMy H-modes is still

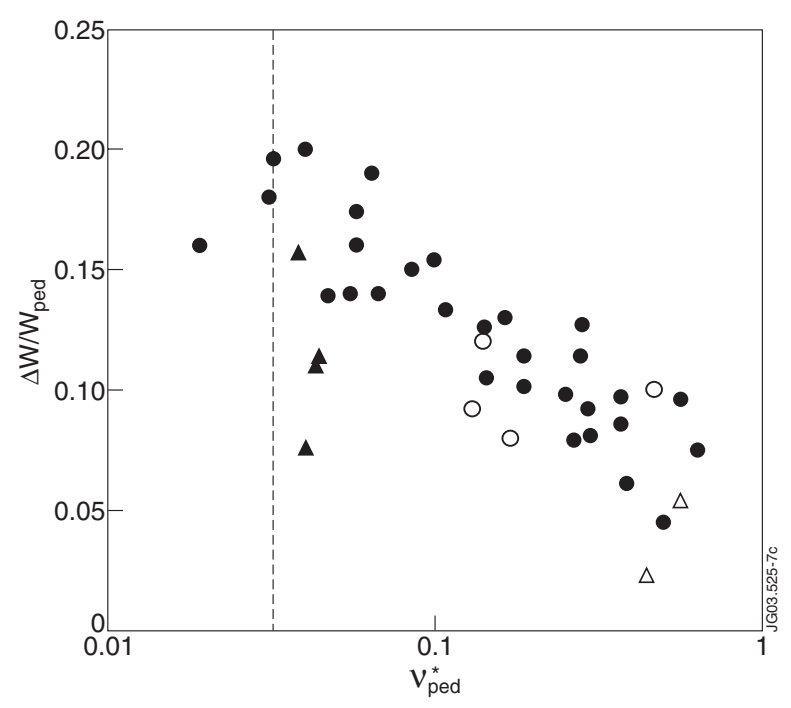

Figure 13. Comparison of type-III ELMy H-modes to type-I ELMy H-modes: plasma energy loss due to ELMs normalized to pedestal stored energy versus pedestal electron collisionality, $v^{*}$; non-seeded type-I ELMy H-modes (๑), seeded type-I ELMy H-modes (O), non-seeded type-III ELMy H-modes $(\boldsymbol{\Delta})$ and seeded type-III ELMy H-modes $(\triangle)$; the ITER collisionality (- - - ). a factor of $\approx 2$ lower than in type-I ELMy H-modes. The real ELM losses may be up to a factor of 2 lower than these estimates, as indicated by kinetic measurements of $\Delta W$ [7] and the simple estimation of the ELM losses in section 2.1, which showed lower values than the kinetic measurements even. For ITER, an ELM size of $\Delta W / W_{\text {ped }}$ of $9 \pm 5 \%$ (assuming $W_{\text {ped }}=30 \% W$ ) seems to be just acceptable $[6,4]$. As shown in figure 14 , the contribution of the pedestal stored energy to the total stored energy is generally lower for type-III ELMs than for type-I ELMs, remaining within the ITER reference of $W_{\text {ped }}=30 \% \mathrm{~W}$ even at high stored energy (and triangularity, up to $\delta \approx 0.5$ ). As is demonstrated in figure 13 , the type-III ELM losses are with $\Delta W / W_{\text {ped }}$ of $11 \pm 4 \%$, for the lowest collisionality, just within the ITER acceptance $\left(\Delta W / W_{\text {ped }}\right.$ of $9 \pm 5 \%$ ), whereas for the ITER edge collisionality, significantly too large type-I ELM losses are observed. However, if the ELM size in ITER is not determined by the edge collisionality but rather by the parallel transport times in the SOL, type-I ELMy H-modes might be just acceptable [4].

Radiating ELMy H-modes also have the advantage of lowering the target surface temperature between ELMs. This allows for higher transient heat flux densities since there is greater headroom between the base temperature and the temperature of carbon ablation $\left(1800^{\circ} \mathrm{C}\right)$, which sets the limit for the transient heat flux.

The optimization of an integrated ITER scenario is difficult. Whereas $\Delta W / W$ and $P_{\text {rad }} / P_{\text {heat }}$ are determined by the power exhaust capabilities of the divertor, the confinement enhancement factor, $\beta_{\mathrm{N}}, q_{95}, n_{\mathrm{e}} / n^{\mathrm{GW}}$ and fuel purity are determined by the condition to meet $Q=10$. Unfortunately, performance optimization and divertor heat load control are not easily combined. Too high radiation is often observed to degrade the confinement too much. Also, increased radiation will obviously reduce the fuel purity. On the other hand, as shown in this article, a reduction in $\Delta W / W$ seems to be only possible by a degradation of the edge pedestal and hence the overall confinement. To illustrate the achievements so far, the best discharges with respect to the ITER requirements are

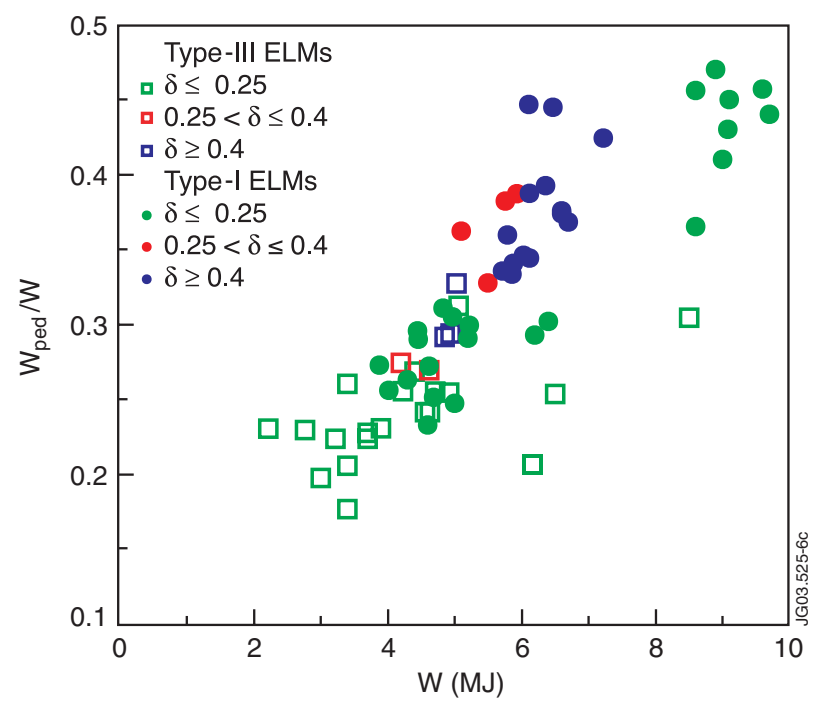

Figure 14. Comparison of type-III ELMy H-modes with type-I ELMy H-modes: ratio of pedestal to total stored energy, $W_{\text {ped }} / W$, versus stored plasma energy, $W$. 
Table 1. Best radiative pulses achieved so far for an integrated ITER scenario: \#55969 and \#59029; the ITER values are taken from $[1,13]$.

\begin{tabular}{llll}
\hline & ITER: & JET: & JET: \\
& $Q=10$ at 17 MA & \#59029 & $\# 55969$ \\
\hline$I_{\mathrm{p}}$ (MA) & 17 & 2.5 & 2.5 \\
$B_{\mathrm{t}}$ (T) & 5.3 & 2.0 & 2.7 \\
$H_{98(y, 2)}$ & 0.75 & 0.73 & 0.74 \\
$f_{\mathrm{GDL}}$ & 1.0 & 1.05 & 1.0 \\
$\beta_{\mathrm{N}}$ & 1.5 & 1.7 & 1.4 \\
$q_{95}$ & 2.6 & 2.6 & 3.0 \\
$f_{\text {rad }}$ & 0.75 & 0.8 & 0.75 \\
$Z_{\text {eff }}$ & 1.7 & 2.2 & 1.6 \\
$\delta$ & 0.5 & 0.44 & 0.47 \\
\hline
\end{tabular}

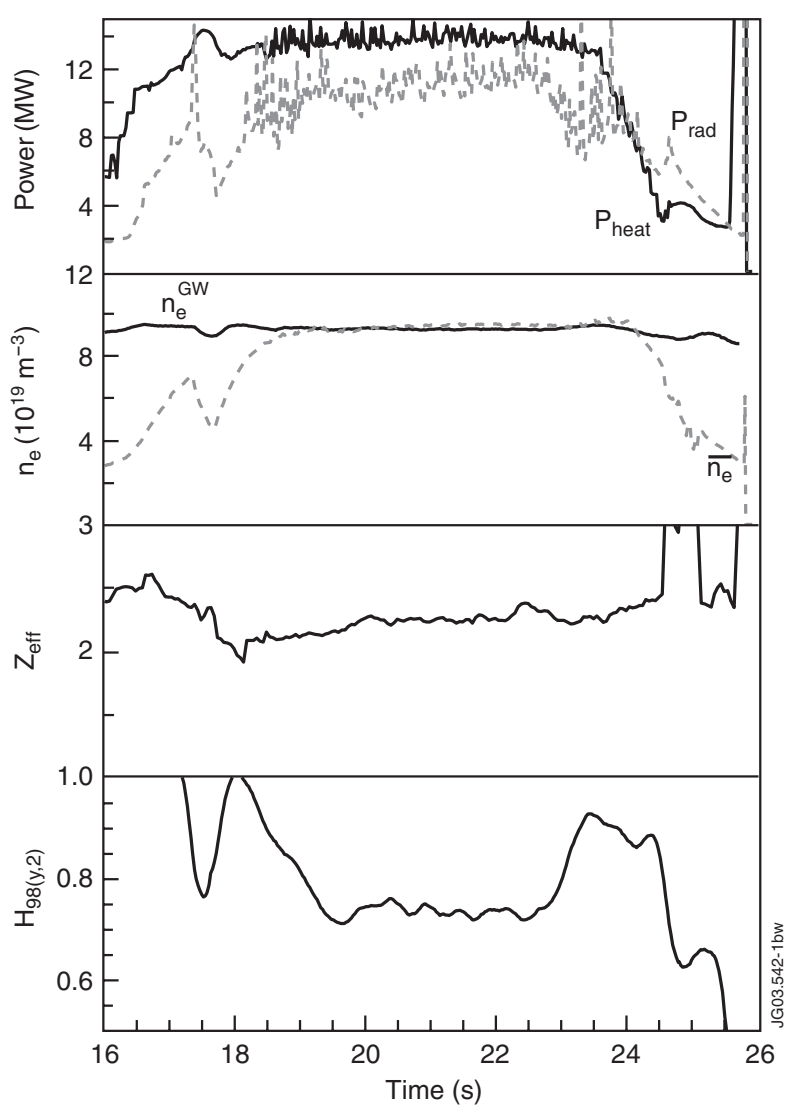

Figure 15. Overview of nitrogen seeded type-III ELMy H-mode \#59029, 2.5 MA, 2.0 T

summarized in table 1 . Since the scaling of the heat load to the divertor as a result of the ELMs is not consolidated yet, it is not listed in this table. Figure 15 shows an overview of a discharge (\#59029) that demonstrates the ability of the radiative type-III ELMy H-mode to meet nearly all the requirements for an integrated ITER scenario.

\section{Conclusions}

By seeding impurities, the inter-ELM heat flux to the divertor target plates can be reduced substantially. Radiative power fractions of up to $90 \%$ did lead to negligible steady state power fluxes to the divertor. For both type-III ELMy H-modes and
type-I ELMy H-modes, a reduction of the transient heat flux was also observed. This reduction of the heat flux is mainly due to a decrease in the pedestal energy in the presence of impurity fuelling. ELM mitigation by radiative dissipation of the ELM energy was only observed for the smallest type-III ELMs, and this has been shown to be consistent with EDGE2D/NIMBUS modelling. However, this radiative dissipation of ELM energy is not expected for ITER since the ELM energy is expected to be in excess of several megajoules. Hence, the ELM energy loss has to be limited. This reduction in ELM size could be achieved in the radiative type-III ELMy $\mathrm{H}$-mode or by a reduction of the pedestal pressure in the radiative type-I ELMy H-mode. It is predicted that for ITER-like pedestal collisionalities, type-III ELMs are acceptable, whereas the minimum type-I ELMs are extremely marginal for surface ablation. However, the uncertainties in extrapolation to ITER are significant, and so both approaches are worthy of further development.

\section{Acknowledgment}

This work has been conducted under the European Fusion Development Agreement.

\section{References}

[1] Campbell D.J. 2001 Phys. Plasmas 82041

[2] Gruber O. et al 1999 Nucl. Fusion 391321

[3] Konoshima S. et al 2001 Plasma Phys. Control. Fusion 43959

[4] Loarte A. et al 2003 J. Nucl. Mater. 313-316 919

[5] Matthews G.F. et al 2003 Nucl. Fusion 43999

[6] Federici G. et al 2003 Plasma Phys. Control. Fusion 451523

[7] Rapp J. et al 2002 Plasma Phys. Control. Fusion 44639

[8] Kallenbach A. et al 1995 Nucl. Fusion 351231

[9] Suttrop W. et al 1997 Plasma Phys. Control. Fusion 392051

[10] Saibene G. et al 1999 Nucl. Fusion 391133

[11] Gruber O. et al 1997 Plasma Phys. Control. Fusion 39 B19

[12] Saibene G. 2002 Plasma Phys. Control. Fusion 441769

[13] Shimada M. et al 2000 J. Plasma Fusion Res. Ser. 377

[14] Horton L.D. et al 1999 Nucl. Fusion 39993

[15] Dumortier P. et al 2002 Plasma Phys. Control. Fusion 441845

[16] De La Luna E. et al 2003 Rev. Sci. Instrum. 741414

[17] Kempenaars M. et al 2003 Proc. 30th European Physical Society Conf. on Controlled Fusion and Plasma Physics (St Petersburg, Russia, 7-11 July 2003) vol 27a (Europhysics Conference Abstracts) (Geneva: European Physical Society) P-1.56

[18] Eich T. et al 2003 J. Nucl. Mater. 313-316 919

[19] Jachmich S. et al 2002 Plasma Phys. Control. Fusion 441879

[20] Suttrop W. et al 2002 Phys. Plasmas 92103

[21] Tokar M.Z. et al 1997 Nucl. Fusion 371691

[22] Nave M.F.F. et al 2003 Nucl. Fusion 431204

[23] Simonini et al 1994 Contrib. Plasma Phys. 44368

[24] Kallenbach A. et al 2002 Proc. 29th European Physical Society Conf. on Controlled Fusion and Plasma Physics (Montreux, Switzerland, June 2002) vol 26b (Europhysics Conference Abstracts) (Geneva: European Physical Society) O-3.22

[25] Loarte A. et al 2000 IAEA-CN-77 ITERP/11(R) 18th IAEA Conf. (Sorrento, Italy, 4-10 October 2000) Proc. 18th Int. Conf. on Fusion Energy 2000 (Sorrento, 2000) (Vienna: IAEA) CD-ROM file ITERP_411.pdf and http://www.iaeaorg/ programmes/ripc/physics/fec2000/html/node1.htm

[26] Loarte A. et al 2003 Plasma Phys. Control. Fusion 491549

[27] Fishpool G.M. 1998 Nucl. Fusion 381373 